\title{
A NONLINEAR THEORY FOR SHELLS WITH SLOWLY VARYING THICKNESS
}

\author{
MARTA LEWICKA, MARIA GIOVANNA MORA AND MOHAMMAD REZA PAKZAD
}

\begin{abstract}
We study the $\Gamma$-limit of $3 \mathrm{~d}$ nonlinear elasticity for shells of small, variable thickness, around an arbitrary smooth $2 \mathrm{~d}$ surface.
\end{abstract}

\section{INTRODUCTION}

The following question receives large attention in the current literature on elasticity [1]: which theories of thin objects (rods, plates, shells) are predicted by the $3 \mathrm{~d}$ nonlinear theory? For plates, this problem has been extensively studied through $\Gamma$-convergence; first by LeDret and Raoult [5], leading to a rigorous derivation of the membrane theory, and later by Friesecke, James and Müller [4, for the Kirchhoff, von Kármán and linear theories. In this framework, much less has been done for shells. The membrane and the bending theories were obtained in [6] and [2], respectively. More recently, the generalized von Kármán and linear theories have been rigorously introduced and justified by the authors in 7 . In this paper, we present these last new results, in an extended version for shells with variable thickness and mid-surface.

\section{The SETting of THE PROBLEM AND THE RELATED ELASTIC ENERGy} FUNCTIONALS

The three-dimensional problem. Let $S$ be a $2 \mathrm{~d}$ compact, connected, oriented surface in $\mathbb{R}^{3}$, of class $\mathcal{C}^{1,1}$, whose boundary is the union of finitely many (possibly none) lipschitz curves. Let $\vec{n}(x)$ be the unit normal vector, $T_{x} S$ the tangent space, and $\Pi(x)=\nabla \vec{n}(x)$ the shape operator on $S$, at a given $x \in S$.

For given positive lipschitz functions $g_{1}, g_{2}: S \longrightarrow(0, \infty)$, small in $L^{\infty}$, consider a family of thin shells:

$$
S^{h}=\left\{z=x+t \vec{n}(x) ; x \in S,-h g_{1}(x)<t<h g_{2}(x)\right\}, \quad 0<h<1 .
$$

The elastic energy (scaled per unit thickness) of a deformation $u^{h} \in W^{1,2}\left(S^{h}, \mathbb{R}^{3}\right)$ is given by:

$$
E^{h}\left(u^{h}\right)=\frac{1}{h} \int_{S^{h}} W\left(\nabla u^{h}\right),
$$

where the stored-energy density $W: \mathbb{R}^{3 \times 3} \longrightarrow[0, \infty]$ is $\mathcal{C}^{2}$ in a neighborhood of $S O(3)$, and satisfies:

$$
\begin{aligned}
\forall F \in \mathbb{R}^{3 \times 3} \quad \forall R \in S O(3) \quad W(R)=0, \quad W(R F)=W(F), \\
\\
W(F) \geq C \operatorname{dist}^{2}(F, S O(3)) .
\end{aligned}
$$

1991 Mathematics Subject Classification. 74K20, 74B20.

Key words and phrases. shell theories, nonlinear elasticity, Gamma convergence, calculus of variations. 
The two-dimensional problem. We now introduce the von Kármán functional on $S$ :

$$
\begin{gathered}
I\left(V, B_{t a n}\right)=\frac{1}{2} \int_{S}\left(g_{1}+g_{2}\right) \mathcal{Q}_{2}\left(x, B_{t a n}-\frac{\kappa}{2}\left(A^{2}\right)_{t a n}-\frac{1}{2} \operatorname{sym}\left(A \nabla\left(\left(g_{2}-g_{1}\right) \vec{n}\right)\right)\right) \\
+\frac{1}{24} \int_{S}\left(g_{1}+g_{2}\right)^{3} \mathcal{Q}_{2}\left(x,(\nabla(A \vec{n})-A \Pi)_{t a n}\right),
\end{gathered}
$$

defined for $V \in \mathcal{V}$ and $B_{\text {tan }} \in \mathcal{B}$. The space $\mathcal{V}$ consists of infinitesimal isometries $V \in W^{2,2}\left(S, \mathbb{R}^{3}\right)$, that is these vector fields $V$ for whom there exists a matrix field $A \in W^{1,2}\left(S, \mathbb{R}^{3 \times 3}\right)$ so that:

$$
\partial_{\tau} V(x)=A(x) \tau \quad \text { and } \quad A(x)^{T}=-A(x) \quad \forall \text { a.e. } x \in S \quad \forall \tau \in T_{x} S .
$$

The finite strain space $\mathcal{B}$ (see [7]), consists of the following symmetric matrix fields:

$$
\mathcal{B}=\left\{L^{2}-\lim _{h \rightarrow 0} \operatorname{sym} \nabla w^{h} ; w^{h} \in W^{1,2}\left(S, \mathbb{R}^{3}\right)\right\} .
$$

In (2.2) $\kappa \geq 0$ is a parameter, and the positive definite quadratic forms $\mathcal{Q}_{2}(x, \cdot)$ are defined as follows:

$$
\mathcal{Q}_{2}\left(x, F_{\text {tan }}\right)=\min \left\{\mathcal{Q}_{3}(\tilde{F}) ;(\tilde{F}-F)_{\text {tan }}=0\right\}, \quad \mathcal{Q}_{3}(F)=D^{2} W(\mathrm{Id})(F, F) .
$$

The quadratic form $\mathcal{Q}_{3}$ is defined for all $F \in \mathbb{R}^{3 \times 3}$, while $\mathcal{Q}_{2}(x, \cdot)$, for a given $x \in S$ is defined on tangential minors $F_{t a n}$ of such matrices. Recall that the tangent space to $S O(3)$ at Id is $s o(3)$. As a consequence, both forms depend only on the symmetric parts of their arguments and are positive definite on the space of symmetric matrices $[3]$.

Relative stretching and bending. The two terms in (2.2) are strictly tied to the deformations of the geometric mid-surface of $S^{h}$. Given $V \in \mathcal{V}$ and a field $w \in W^{1,2}\left(S, \mathbb{R}^{3}\right)$, consider the deformations:

$$
\tilde{\phi}^{h}=\mathrm{id}+1 / 2 h\left(g_{2}-g_{1}\right) \vec{n}, \quad \phi^{h}=\tilde{\phi}^{h}+h V+h^{2} w .
$$

Then $\tilde{\phi}^{h}(S)$ is the geometric mid-surface. A straightforward calculation shows that for any $\tau \in T_{x} S$ :

$\left|\partial_{\tau} \phi^{h}\right|^{2}-\left|\partial_{\tau} \tilde{\phi}^{h}\right|^{2}=2 h^{2} \tau^{T}\left(\operatorname{sym} \nabla w-1 / 2 A^{2}-1 / 2 \operatorname{sym}\left(A \nabla\left(\left(g_{2}-g_{1}\right) \vec{n}\right)\right)\right) \tau+\mathcal{O}\left(h^{3}\right)$.

Hence (putting $\kappa=1$ and $B_{\text {tan }}=\operatorname{sym} \nabla w$ ), the expression in the argument of $\mathcal{Q}_{2}$ in the first term of (2.2) describes stretching, namely the second order in $h$ change of the first fundamental form of $\tilde{\phi}^{h}(S)$.

The second term of (2.2) relates to bending, that is the first order in $h$ change in the second fundamental form of $\tilde{\phi}^{h}(S)$. To see this, let $\Pi^{h}$ be the shape operator on $\phi^{h}(S)$. Indeed, for $\tau \in T_{x} S$ one obtains:

$$
\left(\nabla \phi^{h}\right)^{-1} \Pi^{h}\left(\nabla \phi^{h}\right) \tau-\left(\nabla \tilde{\phi}^{h}\right)^{-1} \Pi\left(\nabla \tilde{\phi}^{h}\right) \tau=h\left(\partial_{\tau}(A \vec{n})-A \Pi \tau\right)+\mathcal{O}\left(h^{2}\right) .
$$

Notice also, that the term $-1 / 2 \operatorname{sym}\left(A \nabla\left(\left(g_{2}-g_{1}\right) \vec{n}\right)\right)$ which is new with respect to analysis in [7, disappears when $g_{1}=g_{2}$ or equivalently $S=\tilde{\phi}^{h}(S)$. This term expresses the first order in $h$ deficit for $V$ from being an infinitesimal isometry on $\tilde{\phi}^{h}(S)$. It is because for any $\eta=\partial_{\tau} \tilde{\phi}^{h}(x)$ where $\tau \in T_{x} S$ we have:

$$
\partial_{\eta}\left(V \circ\left(\tilde{\phi}^{h}\right)^{-1}\right) \cdot \eta=\partial_{\tau} V \cdot \partial_{\tau} \tilde{\phi}^{h}=-h / 2 \tau^{T} \operatorname{sym}\left(A \nabla\left(\left(g_{2}-g_{1}\right) \vec{n}\right)\right) \tau .
$$




\section{The $\Gamma$-CONVERGENCE OF THE ELASTIC ENERGY FUNCTIONALS}

Theorem 3.1. Let $e^{h}$ be a sequence of positive numbers, for which we assume:

$$
\lim _{h \rightarrow 0} e^{h} / h^{4}=\kappa^{2}<\infty .
$$

(a) For any sequence of deformations $u^{h} \in W^{1,2}\left(S^{h}, \mathbb{R}^{3}\right)$ satisfying:

$$
E^{h}\left(u^{h}\right) \leq C e^{h},
$$

there exist a sequence $Q^{h} \in S O(3)$ and $c^{h} \in \mathbb{R}^{3}$ such that for the normalized rescaled deformations: $y^{h}(x+t \vec{n})=\left(Q^{h}\right)^{T} u^{h}(x+h t \vec{n})-c^{h}$ defined on the common domain $S^{1}$, the following holds.

(i) $y^{h}$ converge in $W^{1,2}\left(S^{1}\right)$ to the projection $\pi: S^{1} \longrightarrow S$ along the normal $\vec{n}$.

(ii) The related scaled average displacements:

$$
\left(V^{h}\left[y^{h}\right]\right)(x)=\frac{h}{\sqrt{e^{h}}} f_{-g_{1}(x)}^{g_{2}(x)} y^{h}(x+t \vec{n})-(x+h t \vec{n}) d t
$$

converge (up to a subsequence) in $W^{1,2}(S)$ to some $V \in \mathcal{V}$.

(iii) $\frac{1}{h} \operatorname{sym} \nabla V^{h}\left[y^{h}\right]$ converge (up to a subsequence) weakly in $L^{2}(S)$ to some $B_{\text {tan }} \in \mathcal{B}$.

(iv) $\liminf _{h \rightarrow 0} \frac{1}{e^{h}} E^{h}\left(u^{h}\right) \geq I\left(V, B_{\text {tan }}\right)$.

(b) Conversely, for every $V \in \mathcal{V}$ and $B_{\text {tan }} \in \mathcal{B}$, there exists a sequence $u^{h} \in$ $W^{1,2}\left(S^{h}, \mathbb{R}^{3}\right.$ ) satisfying (3.2) and such that (i), (ii), (iii) hold (with $Q^{h}=\operatorname{Id}$ and $\left.c^{h}=0\right)$ and moreover: $\lim _{h \rightarrow 0} \frac{1}{e^{h}} E^{h}\left(y^{h}\right)=I\left(V, B_{\text {tan }}\right)$.

Proof. Here we outline the main steps of the proof of Theorem 3.1. We refer the readers to [7] for a detailed exposition in the case $g_{1}=g_{2}=$ const. All convergences below are up to a subsequence.

1. A first major ingredient is approximating $u^{h}$ by $W^{1,2}$ matrix fields $R^{h}: S \rightarrow$ $S O(3)$, thanks to the scaling invariant nonlinear quantitative rigidity estimate from [3. Noting (3.2) and the uniform equivalence of the functionals $\int \operatorname{dist}^{2}(\nabla \cdot, S O(3))$ and $E^{h}(\cdot)$ on the uniformly lipschitz domains $S^{h}$, it follows that:

$\left\|\nabla u^{h}-R^{h} \pi\right\|_{L^{2}\left(S^{h}\right)} \leq C h^{1 / 2} \sqrt{e^{h}}, \quad\left\|\nabla R^{h}\right\|_{L^{2}(S)}+\left\|R^{h}-Q^{h}\right\|_{L^{2}(S)} \leq C h^{-1} \sqrt{e^{h}}$

for some $Q^{h} \in S O(3)$. Further, for some skew-symmetric matrix field $A \in W^{1,2}\left(S, \mathbb{R}^{3}\right)$, one has:

$$
\begin{aligned}
& h / \sqrt{e^{h}}\left(\left(Q^{h}\right)^{T} R^{h}-\mathrm{Id}\right) \longrightarrow A \quad \text { weakly in } W^{1,2}(S), \\
& h^{2} / e^{h} \operatorname{sym}\left(\left(Q^{h}\right)^{T} R^{T}-\mathrm{Id}\right) \longrightarrow 1 / 2 A^{2} \quad \text { strongly in } L^{2}(S) .
\end{aligned}
$$

2. Define now:

$$
w^{h}(x+t \vec{n})=h / \sqrt{e^{h}}\left(\left(Q^{h}\right)^{T} u^{h}(x+h t \vec{n})-c^{h}-(x+h t \vec{n})\right),
$$

with $c^{h}$ such that $\int_{S^{1}} w^{h}=0$. To prove (i) and (ii), one first uses the above convergences to obtain that:

$$
\nabla_{t a n} w^{h} \longrightarrow A \pi(\operatorname{Id}+t \Pi)^{-1} \quad \text { and } \quad \partial_{\vec{n}} w^{h} \longrightarrow 0, \quad \text { strongly in } L^{2}\left(S^{1}\right) .
$$


By the Poincaré inequality, $w^{h}$ must now converge to $V \circ \pi$, strongly in $W^{1,2}\left(S^{1}\right)$.

For (iii), one equates various terms in $\frac{1}{h} \operatorname{sym} \nabla V^{h}\left[y^{h}\right]$ to notice that it converges weakly in $L^{2}(S)$ to:

$$
f_{-g_{1}}^{g_{2}}(\operatorname{sym} G)_{t a n} \mathrm{~d} t+\frac{\kappa}{2}\left(A^{2}\right)_{t a n}+\frac{1}{2} \operatorname{sym}\left(A \nabla\left(\left(g_{2}-g_{1}\right) \vec{n}\right)\right)=: B_{\text {tan }},
$$

where $G$ is the weak $L^{2}\left(S^{1}\right)$ limit of the matrix fields:

$$
G^{h}(x+t \vec{n})=\frac{1}{\sqrt{e^{h}}}\left(\left(R^{h}\right)^{T} \nabla u^{h}(x+h t \vec{n})-\mathrm{Id}\right) .
$$

The convergence of $G^{h}$ [7, which is the $\sqrt{e^{h}}$ order term in the expansion of the nonlinear strain $\left(\nabla u^{h}\right)^{T} \nabla u^{h}$ at Id, plays a major role for the expansion of the energy density: $W\left(\nabla u^{h}\right)=W\left(\operatorname{Id}+\sqrt{e^{h}} G^{h}\right)$.

3. Towards proving (iv), the previously established convergences and the definition of $\mathcal{Q}_{2}$ imply:

$$
\begin{aligned}
& \liminf _{h \rightarrow 0} \frac{1}{e^{h}} E^{h}\left(u^{h}\right) \geq \frac{1}{2} \int_{S} \int_{-g_{1}}^{g_{2}} \mathcal{Q}_{2}\left(x,(\operatorname{sym} G)_{t a n}\right) \mathrm{d} t \mathrm{~d} x \\
& =\frac{1}{2} \int_{S} \int_{-\left(g_{1}+g_{2}\right) / 2}^{\left(g_{1}+g_{2}\right) / 2} \mathcal{Q}_{2}\left(x,\left(\operatorname{sym} G_{0}\right)_{t a n}+\left(s+\left(g_{2}-g_{1}\right) / 2\right)\left(\nabla_{t a n} A\right) \vec{n}\right) \mathrm{d} s \mathrm{~d} x,
\end{aligned}
$$

where we used the fact that:

$$
(\operatorname{sym} G)_{\tan }(x+t \vec{n})=\left(\operatorname{sym} G_{0}\right)_{\tan }(x)+t\left(\nabla_{t a n} A\right) \vec{n}
$$

is linear in $t$. Calculating ( $\left.\operatorname{sym} G_{0}\right)_{\text {tan }}$ from (3.3) and collecting various terms in the argument of the quadratic form $\mathcal{Q}_{2}$ above, we see that the right hand side in (3.4) equals the von Kármán functional $I\left(V, B_{t a n}\right)$.

4. If $V \in \mathcal{V} \cap W^{2, \infty}\left(S, \mathbb{R}^{3}\right)$ and $B_{\text {tan }}=\operatorname{sym} \nabla w$ with $w \in W^{2, \infty}\left(S, \mathbb{R}^{3}\right)$, then the recovery sequence is given by the formula below. When $V$ and $B_{\text {tan }}$ do not have the required regularity, one proceeds by approximation and truncation, as in [7].

$$
\begin{aligned}
y^{h}(x+t \vec{n}) & =x+h / 2\left(g_{2}-g_{1}\right) \vec{n}(x)+\sqrt{e^{h}} / h V(x)+\sqrt{e^{h}} w(x) \\
+ & h\left(t-1 / 2\left(g_{2}-g_{1}\right)\right) \vec{n}(x)+\left(t-1 / 2\left(g_{2}-g_{1}\right)\right) \sqrt{e^{h}}\left(\Pi V_{\text {tan }}-\nabla(V \vec{n})\right)(x) \\
& -h\left(t-1 / 2\left(g_{2}-g_{1}\right)\right) \sqrt{e^{h}} \vec{n}^{T} \nabla w+\left(t-1 / 2\left(g_{2}-g_{1}\right)\right) h \sqrt{e^{h}} d^{0}(x) \\
+ & 1 / 2\left(t-1 / 2\left(g_{2}-g_{1}\right)\right)^{2} h \sqrt{e^{h}} d^{1}(x) .
\end{aligned}
$$

The vector fields $d^{0}, d^{1} \in W^{1, \infty}\left(S, \mathbb{R}^{3}\right)$ are given by means of the linear map $c\left(x, F_{\text {tan }}\right)$ which returns the unique vector $c$ satisfying:

$$
\mathcal{Q}_{2}\left(x, F_{\text {tan }}\right)=\min \left\{\mathcal{Q}_{3}\left(F_{\text {tan }}+c \otimes \vec{n}(x)+\vec{n}(x) \otimes c\right) ; c \in \mathbb{R}^{3}\right\} .
$$

Then:

$$
\begin{gathered}
d^{0}=2 c\left(x, B_{\text {tan }}-\frac{\kappa}{2}\left(A^{2}\right)_{\text {tan }}-\frac{1}{2} \operatorname{sym}\left(A \nabla\left(\left(g_{2}-g_{1}\right) \vec{n}\right)\right)\right) \\
+\kappa A^{2} \vec{n}-\frac{\kappa}{2}\left(\vec{n}^{T} A^{2} \vec{n}\right) \vec{n}+\frac{1}{2}\left(A \nabla\left(\left(g_{2}-g_{1}\right) \vec{n}\right)\right)^{T} \vec{n} \\
d^{1}=2 c\left(x, \operatorname{sym}(\nabla(A \vec{n})-A \Pi)_{\tan }\right)+\vec{n}^{T} A \Pi-\vec{n}^{T} \nabla(A \vec{n}) .
\end{gathered}
$$




\section{THE DEAD LOADS AND CONVERGENCE OF MINIMIZERS}

Consider a sequence of forces $f^{h} \in L^{2}\left(S, \mathbb{R}^{3}\right)$ acting on $S$, with the following properties:

$$
\int_{S}\left(g_{1}+g_{2}\right) f^{h}=0 \quad \text { and } \quad \lim _{h \rightarrow 0} \frac{1}{h \sqrt{e^{h}}} f^{h}=f \quad \text { weakly in } L^{2}(S) .
$$

Define their extensions $f^{h}(x+t \vec{n})=\operatorname{det}(\operatorname{Id}+t \Pi(x))^{-1} f^{h}(x)$ over $S^{h}$, and the maximized action of $f^{h}$ over all rotations of $S^{h}$ as:

$$
m^{h}=\max _{Q \in S O(3)} \frac{1}{h} \int_{S^{h}} f^{h}(z) \cdot Q z \mathrm{~d} z .
$$

The total energy functional on $S^{h}$ is:

$$
J^{h}\left(u^{h}\right)=E^{h}\left(u^{h}\right)+m^{h}-\frac{1}{h} \int_{S^{h}} f^{h} u^{h} .
$$

As in [7, one can prove that:

$$
0 \geq \inf \left\{\frac{1}{e^{h}} J^{h}\left(y^{h}\right) ; u^{h} \in W^{1,2}\left(S^{h}, \mathbb{R}^{3}\right)\right\} \geq-C .
$$

We further introduce the relaxation function $r: S O(3) \longrightarrow[0, \infty]$, with its effective domain $\mathcal{M}=\{\bar{Q} \in S O(3) ; r(\bar{Q})<\infty\}$ :

$$
r(Q)=\inf \left\{\liminf _{h \rightarrow 0} \frac{1}{e^{h}}\left(m^{h}-\frac{1}{h} \int_{S^{h}} f^{h} \cdot Q^{h} z \mathrm{~d} z\right) ; Q^{h} \in S O(3), Q^{h} \rightarrow Q\right\}
$$

Theorem 4.1. Assume (3.1) and (4.1). Let $u^{h} \in W^{1,2}\left(S, \mathbb{R}^{3}\right)$ be any minimizing sequence of $\frac{1}{e^{h}} J^{h}$, that is:

$$
\lim _{h \rightarrow 0}\left(\frac{1}{e^{h}} J^{h}\left(y^{h}\right)-\inf \frac{1}{e^{h}} J^{h}\right)=0,
$$

Then the conclusions of Theorem [3.1 (a) hold, and any accumulation point $\bar{Q}$ of $\left\{Q^{h}\right\}$ belongs to $\mathcal{M}$. Moreover, any limit $\left(V, B_{\text {tan }}, \bar{Q}\right)$ minimizes the following functional, over $V \in \mathcal{V}, B_{\text {tan }} \in \mathcal{B}$ and $\bar{Q} \in \mathcal{M}$ :

$$
J\left(V, B_{t a n}, \bar{Q}\right)=I\left(V, B_{t a n}\right)-\int_{S}\left(g_{1}+g_{2}\right) f \cdot \bar{Q} V+r(\bar{Q}) .
$$

The proof follows as in [7]. An equivalent formulation of Theorem 4.1] in terms of $\Gamma$-convergence is possible.

Example. When $f^{h}=h \sqrt{e^{h}} f$ and $g_{1}=g_{2}$, then:

$$
\mathcal{M}=\left\{\bar{Q} \in S O(3) ; \int_{S} f \cdot \bar{Q} x=\max _{Q \in S O(3)} \int_{S} f \cdot Q x\right\}
$$

(in the general case the inclusion $\subset$ is still true, but not the other one). Further, $r \equiv 0$ on $\mathcal{M}$, so the term $r(\bar{Q})$ may be dropped in the definition of $J$. In the general case, both $r$ and $\mathcal{M}$ depend on the asymptotic behavior of the maximizers of the linear functions $S O(3) \ni Q \mapsto \int_{S^{h}} f^{h}(z) \cdot Q z \mathrm{~d} z$.

Approximately robust surfaces and higher scalings. Some classes of surfaces, including surfaces of revolution, developable surfaces with no flat part, and elliptic surfaces, have the ability to recompense the second order stretching (of $\left.\tilde{\phi}^{h}(S)\right)$ introduced by the infinitesimal isometry $V$, through a suitable second order 
displacement. As an example, surfaces with flat parts do not enjoy this property (for any $g_{1}, g_{2}$ ). For such surfaces [7, described by condition:

$$
\left\{A^{2}+\operatorname{sym}\left(A \nabla\left(\left(g_{2}-g_{1}\right) \vec{n}\right)\right) ; V \in \mathcal{V}\right\} \subset \mathcal{B},
$$

the limit elastic energy $I\left(V, B_{t a n}\right)$ simplifies and should be replaced (in Theorems 3.1 and 4.1, regardless of $\kappa \geq 0$ ) by:

$$
\tilde{I}(V)=\frac{1}{24} \int_{S}\left(g_{1}+g_{2}\right)^{3} \mathcal{Q}_{2}\left(x,(\nabla(A \vec{n})-A \Pi)_{\tan }\right) .
$$

When $\kappa=0$ and $g_{1}=g_{2}$, the stretching term is negligible for any $S$ and hence the von Kármán theory again reduces to the linear theory, with the elastic energy given in (4.4). For more discussion, see [7].

\section{ACKNowledgments}

M.L. was partially supported by the NSF grant DMS-0707275. M.G.M. was partially supported by the Italian Ministry of University and Research through the project "Variational problems with multiple scales" 2006 and by GNAMPA through the project "Problemi di riduzione di dimensione per strutture elastiche sottili" 2008.

\section{REFERENCES}

[1] P.G. Ciarlet, Mathematical Elasticity, North-Holland, Amsterdam (2000).

[2] G. Friesecke, R. James, M.G. Mora, S. Müller, Derivation of nonlinear bending theory for shells from three-dimensional nonlinear elasticity by $\Gamma$-convergence, C. R. Math. Acad. Sci. Paris, 336, (2003), no. 8, 697-702.

[3] G. Friesecke, R. James, S. Müller, A theorem on geometric rigidity and the derivation of nonlinear plate theory from three dimensional elasticity, Comm. Pure. Appl. Math., 55, (2002), 1461-1506.

[4] G. Friesecke, R. James, S. Müller, A hierarchy of plate models derived from nonlinear elasticity by $\Gamma$-convergence, Arch. Ration. Mech. Anal., 180, (2006), no. 2, 183-236.

[5] H. Le Dret, A. Raoult, The nonlinear membrane model as a variational limit of nonlinear three-dimensional elasticity. J. Math. Pures Appl., 73, (1995), 549-578.

[6] H. Le Dret, A. Raoult, The membrane shell model in nonlinear elasticity: a variational asymptotic derivation, J. Nonlinear Sci., 6, (1996), 59-84.

[7] M. Lewicka, M.G. Mora, M.R. Pakzad, Shell theories arising as low energy $\Gamma$-limit of 3d nonlinear elasticity, submitted.

[8] M. Spivak, A Comprehensive Introduction to Differential Geometry, Vol V, 2nd edition, Publish or Perish Inc. (1979).

Marta Lewicka, University of Minnesota, Department of Mathematics, 206 Church St. S.E., Minneapolis, MN 55455

Maria Giovanna Mora, Scuola Internazionale Superiore di Studi Avanzati, via Beirut 2-4, 34014 TRIESTE, ITALY

Mohammad Reza Pakzad, University of Pittsburgh, Department of Mathematics, 139 University Place, Pittsburgh, PA 15260

E-mail address: lewicka@math.umn.edu, mora@sissa.it, pakzad@pitt.edu 